\title{
Waste minimization practices at the National University of Costa Rica
}

\author{
J. Mora, J. Sibaja, J. Rodríguez \& C. Salas \\ National University, Costa Rica
}

\begin{abstract}
Hazardous materials are substances that are flammable, corrosive, reactive, toxic, radioactive, poisonous, carcinogenic, or infectious. According to the USEPA (United State Environmental Protection Agency), waste is considered hazardous if it has one or more of USEPA's four hazardous characteristics: flammability, corrosivity, reactivity or toxicity. One important source of chemical waste is higher education facilities such universities, where the chemical waste generated in the laboratories (labs) present at least one of the hazardous characteristics listed by USEPA. In order to determine the quantity of laboratories doing waste minimization practice related with the USEPA pollution hierarchy, an initial diagnostic about waste management at laboratories was conducted. The data gathered were used to start a training to laboratory personnel training about EPAPH (Environmental Protection Agency-Pollution Hierarchy). After training, new diagnostics were performed. The applications of waste minimization practices in the labs were analyzed before and after the training. The most poorly practices performed year by year during the study period were "substitute nonhazardous materials", "chemical treatment" and "distillation". "Redistribute surplus chemical" was performed in 22 labs, 30 labs and 48 labs during the years 2010, 2011 and 2013 respectively as the most common practice. This study showed that training about pollution prevention hierarchy increased the number of labs doing waste minimization practices, prioritizing reduction and prevention from the source. Future research is recommended to define the chemical waste rate generation before and after training as a quantitative indicator of the impact of training sessions upon the overall institutional chemical waste prevention program.
\end{abstract}

Keywords: hazardous materials, chemical waste management, pollution hierarchy, waste reduction and prevention, laboratories, universities, Costa Rica. 


\section{Introduction}

Hazardous materials are substances that are flammable, corrosive, reactive, toxic, radioactive, poisonous, carcinogenic, or infectious [1, 2]. In a general sense, waste that contain these materials or substances are considered hazardous because they present a potential risk to humans and/or the environment. A hazardous waste management plan generally separates waste into three broad groups: radioactive, chemical, and biohazardous $[3,4]$. The chemical waste includes a wide range of material such as discarded commercial chemical products, process waste, wastewater, or any waste generated from the use of chemicals in medical, dental, veterinary and laboratory procedures that has the potential to pose a chemical threat to health, safety and/or the environment, or is chemically hazardous $[5,6]$. According to the USEPA (United State Environmental Protection Agency), a waste is considered hazardous if it has one or more of USEPA's four hazardous characteristics: flammability, corrosivity, reactivity or toxicity [3, 7].

One important source of chemical waste is higher education facilities such universities, where the chemical waste generated in the laboratories present at least one of the hazardous characteristics listed by USEPA [8-10]. Chemical laboratories are the most common type of workplace where a wide variety of chemicals are handled on a routine basis. The quantity of waste generation in universities is smaller comparing with the production in the industrial sector. However, academic laboratories produce relatively small volumes of a variety of waste streams, the exact character of which may not be known in advance. Besides, in most of the cases, universities contain an important numbers of laboratories on campus, each with numerous points of generation (such as multiple laboratory benchtops) that are operated under the supervision of different individuals [9-11]. Some common waste generated on campus include (but not limited to) unused chemicals that are no longer needed, expired chemicals, process waste, broken mercury thermometers, mercury containing devices, heavy metals, spent acids, bases, and solvents which are used in laboratory procedures, oil based paints, aerosol cans, pesticides, oils (motor, cutting, pump, lubricating) and so on $[11,12]$.

Although some regulations have not recognized the laboratory as a special environment for using chemicals, the OSHA (Occupational Safety and Health Administration) laboratory standard specifies that each institution accountable for handling and disposal of chemicals must develop its own CHP (chemical hygiene plan) to prevent and minimize the waste generation. The waste minimization strategy must incorporate a hierarchical approach to waste reduction $[10,13]$. A start point to develop a CHP (in a university campus) is to follow the USEPA EPA-PH (Environmental Protection Agency-Pollution Hierarchy) which contains four principles $(1-$ source reduction, 2-recycling or re-use, 3-treatment and 4 -disposal) for waste management [7, 12, 14]. The EPA-PH emphasizes source reduction at the top of the pyramid and disposal at the bottom (as illustrated in figure 1) $[3,7,14]$. 


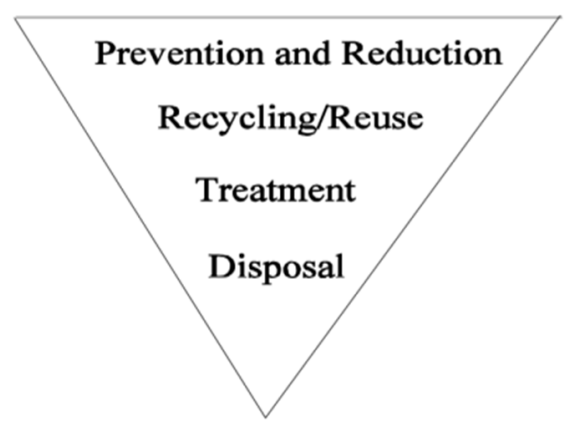

Figure 1: Pollution prevention hierarchy.

The first priority is to reduce waste at the source, with less material entering the waste stream. The familiar concept of recycling, along with composting, is a second priority. A waste is still being generated that must be recycled. It is a better resource management choice to recycle the material than to dispose of it at a landfill. Treatment is a third priority related particularly to hazardous waste. This approach is taken in conjunction with pollution control technologies. Incineration, treatment of sewer discharges, and chemical treatments are included. With solid waste, incineration for energy recovery would be at the same priority level. The lowest priority is land disposal of the final waste stream. This is the most expensive way to use natural resources $[12,14,15]$.

As mentioned before, laboratory operations are notoriously difficult to prescribe waste minimization solutions for. Their waste types are numerous, and usually small in volume. Some waste is extremely hazard to handle due to the need for highly reactive agents in some types of research. These challenges are further compounded in pure research labs where there is a high frequency of change in experimental procedures and reagents used. For this reason, in laboratories, much more so than with industrial operations, it is critical to have a strategy for waste minimization and pollution prevention techniques in use $[7,10]$. According to this, the main objective of this research was to diagnose the quantity of laboratories doing EPA-PH procedures before and after personnel laboratory training. The results are emphasized in the number of sites applying the first, second and third priorities of EPA-PH which are "prevention and reduction from source", "recycling and reuse procedures" and "treatment".

\section{Materials and methods}

The NU-CR (National University-Costa Rica) was founded in 1973, which is a public university with 5,000 workers and 22,000 students. An environmental policy which contains commitments and strategies for a better environment and health security was introduced into the university status in year 2003, this policy was a support to create the CMO (Chemical Management Office) in 2006 where efforts are focused to promote an adequate dangerous waste management, an efficient chemical products handling, EH\&S (environmental health and safety) 
programs and so on. NU-CR among others units contains three faculties related with chemical analytical procedures (Natural Science Faculty, Health Faculty and Soil and Forest Faculty). These faculties have different departments where chemical products are handled. Therefore, chemical waste is produced. The university departments are related with the next science fields: chemistry, biology, agricultural, veterinary, toxicology, environmental, soil and forest. With the main objective to develop waste minimization practices at the laboratories, the CMO started in 2010 a strategy containing the following steps:

- chemical waste management diagnostics;

- diagnostic data analysis;

- laboratory personnel training.

\subsection{Initial chemical waste management diagnostic}

During first semester of year 2010 a first diagnostic regarding waste management in academic instructional and research laboratories was carried out. In order to gather such information, the following tools were utilized: (1) an interview, a questionnaire (personnel of different laboratories fulfilled a form where they were asked information related with chemical waste management); (2) an inspection visit to the laboratories; (3) work sessions with university management authorities; (4) consultation with stakeholders/industry.

\subsection{Data analysis}

The information gathered during diagnostics was analyzed by the CMO with chemical waste experts in order to identify in a general way the following options:

- Reducing the scale experiments conducted in teaching laboratories.

- Replacing some wet laboratories with computer modeling or some analytical manual procedures for automatic equipment procedures.

- Improving training programs.

- Purchasing chemicals procedures.

- Shipping and transporting laboratory chemical waste.

- Generating chemical waste inventories.

- Creating some mechanisms, such as: (1) developing a better procurement management, especially avoiding over ordering of hazardous materials; (2) substituting hazardous materials with less hazardous or nonhazardous materials; (3) reducing the scale of experiments and protocols as much as possible to achieve research objectives; (4) redistributing, reusing, and recycling of supplies and reagents; (5) improving waste segregation in order to maximize recovery of materials and treatability of waste; (6) disseminating information about the benefits of laboratory pollution prevention efforts. 


\subsection{Training}

Individual laboratory training as well as general training was applied during half a year period (II Semester of year 2010 and I Semester of year 2011) in order to discuss the data gathered during the first diagnostic. Faculty staff, department directors, general laboratory operators (students and workers), purchasing staff, financial staff and so on were trained about EPA pollution prevention hierarchy; its opportunities to improve the actual chemical waste management and to look forward to applying new procedures that can protect their health and the environment around. The training was specially focused on the first, second and third EPA hierarchy steps. According to the prevention and reduction steps, different work sessions were conducted in order to discuss and give information about waste source reduction for changing practices and processes to reduce or eliminate the generation of hazardous waste. The training was based on the following WMP (waste minimization practices):

- RR (recovery, reuse or recycling);

- RSC (redistribute surplus chemicals);

- CS (computer simulation);

- CT (chemical treatment), not distillation;

- D (distillation);

- CIM (chemical inventory management);

- PC (purchase control);

- SNM (substitute nonhazardous materials);

- PL (purchase less);

- RS (reduce scale);

- $\mathrm{CE} / \mathrm{P}$ (change equipment or procedure).

The quantity of laboratories which were doing the above waste minimization practices was compared before and after the training.

\subsection{Other chemical waste management diagnostics}

Also, from years 2011 to 2014, new diagnostics were carried out in order to compare the data obtained before and after training. The same methodology criteria and tools used during the first one (year 2010) were applied in the last diagnostics. The number of laboratories during the diagnostics was 60 laboratories (Table 1).

\section{Results and discussion}

Table 1 shows the study population during the diagnostics. Work sessions with university authorities and national stakeholders (Health National Office, Environmental National Office, Private Waste Treatment and Disposal Companies and Landfill Operators) were carried in order to have a better understanding of the results obtained from each diagnostic and seek for future integral solutions.

Figures 2 and 3 indicate the number of laboratories realizing specific WMPs throughout the 2010-2014 period. These figures indicate that during the study 
period "CT" and "D" practices were the least applied. However, overall the number of labs performing these practices increased from year to year.

Table 1: Quantity of laboratories during diagnostics (years 2010-2014).

\begin{tabular}{|l|l|l|}
\hline \multirow{4}{*}{$\begin{array}{l}\text { Study } \\
\text { population }\end{array}$} & Faculty & Department (numbers of labs) \\
\cline { 2 - 3 } & Natural Science Faculty & Biology (10), Chemistry (13), Physics (1) \\
\cline { 2 - 3 } & Health Faculty & Veterinary (16) \\
\cline { 2 - 3 } & Soil and Forest Faculty & $\begin{array}{l}\text { Soil (10), Forest (5), Vulcanology (1), } \\
\text { Environmental (2), Toxicological (2) }\end{array}$ \\
\hline Total & 3 & $9(60)$ \\
\hline
\end{tabular}

During 2010, the most frequently applied WMPs were "SNM", "CT" and "D", at that time only 10 laboratories were performing those practices. For the same year the most performed practice was "RSC" at the level of 22 laboratories.

Starting from 2012, subsequent to training carried out during 2010 to 2011, more laboratories were applying waste minimization practices in comparison to those early years; the number of labs performing every type of WMPs notably increased after 2011.

The most utilized practices during 2012 were "RS" and "PL" both performed at 42 labs. For the 2013 and 2014 years the most performed practices were "RSC" and "PC" with 52 and 55 labs, respectively. Likewise, "PC" was the most common practice for 2014.

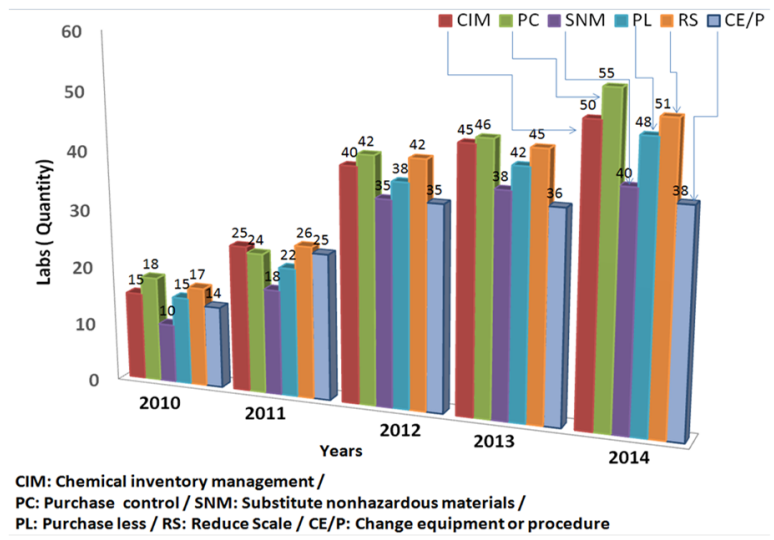

Figure 2: Quantity of laboratories performing six waste minimization practices, period 2010-2014. 


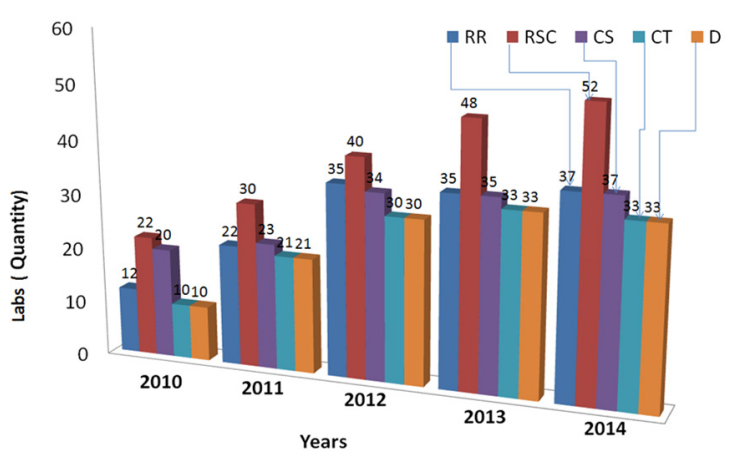

RR: Recovery, reuse or recycling/ RSC: Redistribute Surplus Chemical CS: Computer simulation / CT: Chemical Treatment /D: Distillation

Figure 3: Quantity of laboratories performing five waste minimization practices, period 2010-2014.

Figures 4 and 5 refer to the annual percentage variation for the waste minimization practices evaluated. Practices "CT" and "D" show (Fig. 5) the highest variation (110) throughout the study period. This variation value indicates that when comparing the total number of labs carrying out these practices, 10 labs were doing it during 2010 and 21 labs in 2011. No variation was perceived for "RR" (2013 vs. 2012) Similarly, "CT" and "D" showed no variation (2014 vs. 2013) also standing at 35 total labs. Figure 4, show that "SNM" presented important variations: $80 \%$ (2011 vs. 2010) and 94\% (2012 vs. 2011); nonetheless, variation decreased to $9 \%$ and $5 \%$ in the following years. It can be concluded that starting from 2010 and up to 2012 laboratory operators and directors secured important efforts to substitute hazardous materials.

During 2013 the highest annual percentage variation was only $20 \%$ corresponding to "RSC" (Fig. 5). In the case of 2014 the highest value is assigned to "PC" with a variation of $20 \%$ (Fig. 4).

According to the annual percentage variation graphs and the data relative to the numbers of labs doing specific practices (Figures 2 and 3), the first diagnostic (2010) indicated that "RSC", CS" and "PC" were the most implemented WMPs in 22 labs, 20 labs and 18 labs, respectively. "Substitution with less hazardous or nonhazardous materials also named SNMs", a true source reduction measure, as well as "CT" and "D" were less common, only 10 laboratories used.

Although, the annual variation values surely decreased for the 2013-2014 period, this does not mean a decrease in the quantity of labs carrying out waste minimization practices. The decreasing variation trend only evidences the correct assimilation of waste minimization practices by laboratory personnel as a consequence of the institutional training sessions. 


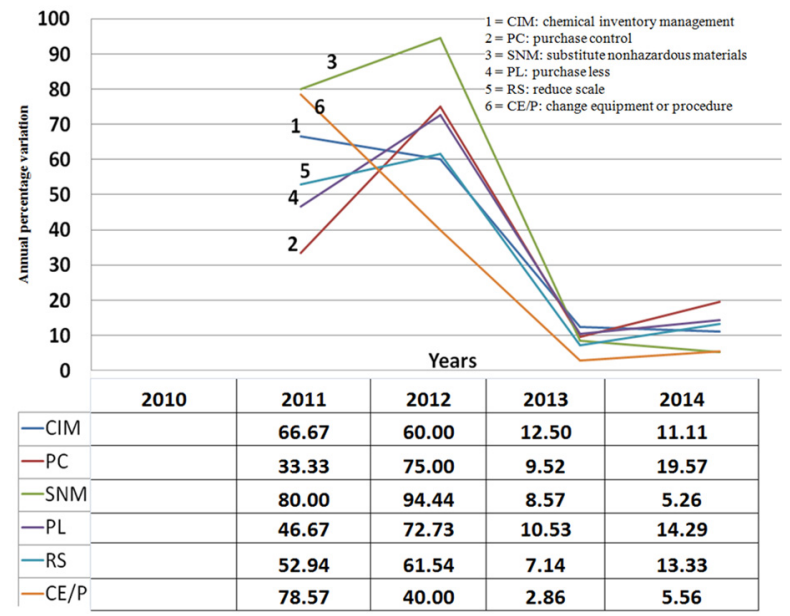

Figure 4: Annual percentage variation for six waste minimization practices, period 2010-2014.

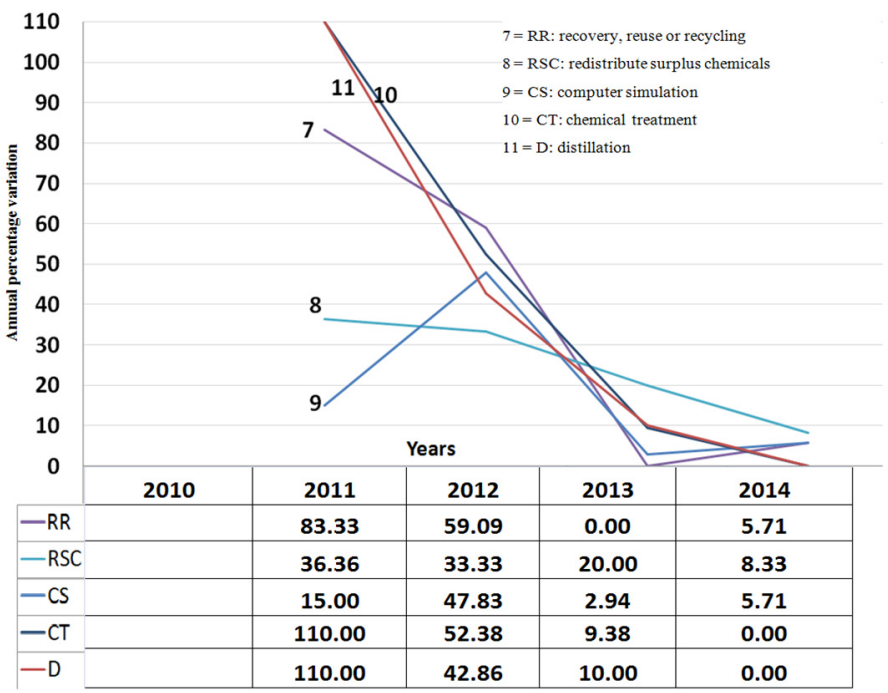

Figure 5: Annual percentage variation of five waste minimization practices, period 2010-2014.

In Figure 6 the black thin line and the indicated numerical value refers to the quantity of labs applying a specific WMP during the year 2014, whereas the thick black bar and the corresponding value represent the differential increase in the quantity of labs performing that same WMP relative to 2010. For example, in the case of "CS", the 17-point differential increase (black thick bar) results from 
practice implementation values going from 20 (arithmetic difference between thin line and thick bar) to 37 (black thin line) for the 2010 and 2014 years, respectively.

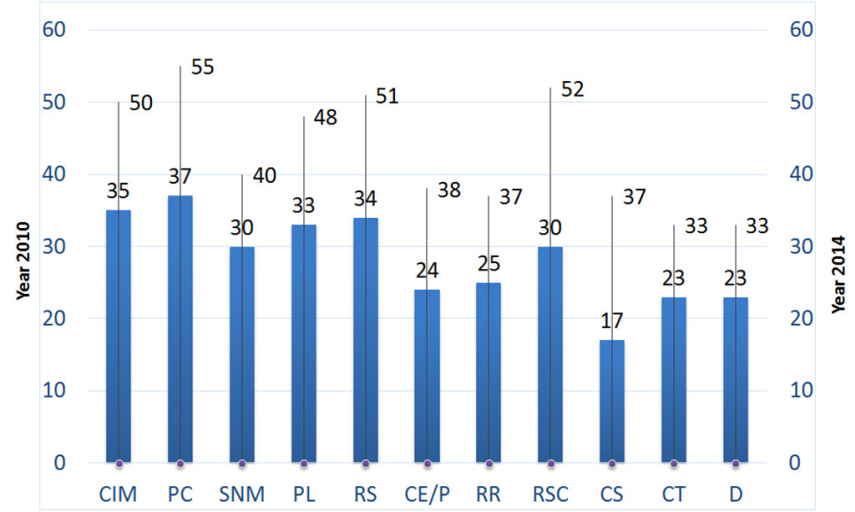

CIM: Chemical inventory management / PC: Purchase control / SNM: Substitute nonhazardous materials / PL: Purchase less / RS: Reduce Scale / CE/P: Change equipment or procedure/RR: Recovery, reuse or recycling/ SC: Redistribute surplus chemicals/ CS: Computer simulation / CT: Chemical Treatment /D: Distillation

Figure 6: Waste minimization practices performed in the years 2010 and 2014.

According to Figure 6, the number of labs applying "CT" and "D" increase from 10 to 33 for each practice. Despite this increase, this quantity is still low considering the total study population (60 labs); this finding is possibly attributed to the lack of available qualified technicians in most of the labs in order to carry out "CT" (neutralization, precipitation, and oxidation-reduction) and distillations ("D") procedures. It is important to point out that according to Table $1,42 \%$ of the labs have chemists as part of their laboratory staff.

Figure 6 indicates that by 2014 most laboratories were actively practicing "CIM" and "PC" which is significant in the sense that these practices inherently facilitate bookkeeping of waste inventory and waste generation rates. "PL" and "RS" were also well implemented. Specifically, the quantity of labs embracing "PL" during 2014 was 48 versus 15 for 2010. Labs operators apply an institutional chemical purchase strategy named "to use and buy just the necessary". It is well to note that a comprehensive "chemical inventory stock" program has been diffused in order to exchange and better redistribute previously unused chemicals within the university community.

In the case of "RS" the 2014 and 2010 figures are 51 and 17, respectively. The evident increase in "RS" practice is in accordance with the global green chemistry initiative which has permeated at the institutional level. For example, a group of researchers at the Department of Chemistry are currently undertaking projects aimed at promoting a shift towards small-scale experiment implementation at the academic laboratory level.

The number of labs implementing "RSC" in 2010 was 22 and 52 in 2014. "CS" is still low ranked with only 10 and 37 out of the 60 total laboratories for 2010 and 2014, respectively. This leaves much room for suggesting the advancement and 
usage of specialized chemical and engineering software at the academic and research level.

Overall, the general trend going from 2010 to 2014 was clearly an increment in the quantity of laboratories implementing WMPs (Fig. 6).

Some specific waste generation prevention and minimization activities completed after training at the UNA-CR are listed below:

- Substitution of ethidium bromide by proprietary nucleic acid stains (Biotium gelred $^{\mathrm{TM}}$ ) for electrophoresis DNA analysis (Veterinary, Biology and Soil and Forest Departments).

- Substitution of acetamide by stearic acid for freezing-point depression determinations in organic chemistry student laboratory practices (Chemistry Department).

- Substitution of alcohol for benzene and lauryl peroxide for benzoyl peroxide as polymer catalysts (Chemistry Department).

- Substitution of mercury containing Kjeldahl digestion catalysts for mercuryfree copper and potassium based catalysts. (Chemistry Department).

- Replacement of toluene by simple alcohols and ketones in organic chemistry laboratory practices (Chemistry Department).

- Replacement of sodium dichromate for sodium hypochlorite as oxidant in various inorganic chemistry laboratory practices (Chemistry Department).

- Usage of peracetic acid and ethanol as an animal cadaver conservative instead of formaldehyde/formalin (Veterinary Department).

- Use of secondary waste containers for chemical waste storage in laboratories.

- Use of non-halogenated solvents instead of halogenated solvents.

- Application of a "Green Chemistry Laboratory Manual" in some chemistry laboratory practices.

- Be wary of receiving external donations of chemicals, accepting only the chemicals that are to be used within the following 3 months.

- Substitution of automatic analysis procedures for manual wet-chemistry procedures, e.g., nitrates by Flow Injection Analysis (FIA) instead of the traditional preparative cadmium column method.

- Use of spent solvent for initial glassware cleaning and fresh solvent for final cleaning.

- Implementation of microscale COD (chemical oxygen demand) analysis methods.

- Mandating the reduction of using reagents containing various toxic metals such as arsenic, barium, cadmium, chromium, lead, mercury, selenium and silver.

- Procurement of state-of-the-art equipment enabling procedures requiring less consumables thus less waste generation.

- Avoiding the use of chromic acid in cleaning solutions and prioritizing the use of detergents, alcohol or just hot water.

- Reusage and recycling of methanol.

- Centralized chemical purchases via the "Chemical Products Office". 
- Avoiding generating "unknown" waste by adhering to proper labeling procedures.

\section{Conclusions}

During this research, the study population comprised 60 institutional laboratories involved in diverse academic and research fields such as biology, toxicology, veterinary, physics, chemistry, environmental sciences, vulcanology and soil and forest sciences.

The less frequently implemented WMPs on a year by year basis (2010-2014) were: "SNM", "CT" and "D".

Redistribute surplus chemicals ("RSC") is the most common practice at a level of 22 labs, 30 labs and 48 labs during the years 2010, 2011 and 2013, respectively. During 2012 "RS" was performed in 42 labs; "PL" was practiced in 55 labs in 2014 , both being the most common WMPs for those two years.

The highest annual percentage variation was evidenced in the 2010-2011 period, right before the institutional waste minimization training process. Although the number of labs performing the considered WMPs always increased year by year, the annual percentage variations evidenced in the 2013-2014 period was less than for the 2010-2011.

Waste minimization practices performed as of 2010 were substantially improved after training, especially the following 2 years. Nonetheless, as of 2014 there are still some low-ranked WMPs such as "CT" and "CP" which should be incentivized by expert training along with financial support for acquiring software technologies.

The least performed WMP for the 2010-2014 period was "CS" with a differential frequency of 17 for this 4-year time period. The highest difference corresponds to "PC" with a difference of 37 labs when comparing 2010 and 2014.

As a general conclusion there are significant obstacles to implementing pollution prevention in universities; initial capital requirements, regulatory barriers, immediate production concerns, lack of staff with technical expertise and institutional inertia. As an initial step towards running successful institutional pollution prevention program a diagnostic of the chemical waste minimization practices was undertaken by highly trained experts of the Chemical Products Office. This office together with university authorities, laboratory managers, principals, investigators, students and technicians look forward to implementing simple and inexpensive solutions aimed at chemical waste minimization and overall pollution prevention.

This study showed that emphasizing pollution prevention hierarchy in institutional training sessions increased the number of laboratories implementing WMPs. Currently, the university has a better and more efficient waste management program which prioritizes waste reduction and prevention from the source.

Future research is recommended to define the chemical waste rate generation before and after training as a quantitative indicator of the impact of training sessions upon the overall institutional chemical waste prevention program. 


\section{References}

[1] Higgins, T., Hazardous Waste Minimization Handbook, Lewis Publishers: Chelsea, 1989.

[2] Lunn, G. and Sansone, E. B., Destruction of Hazardous Chemicals in the Laboratories, 2nd ed., John Wiley \& Sons: England, 1994.

[3] Freeman, H. M., Hazardous Waste Minimization, McGraw-Hill: New York, 1990.

[4] Monz, D. J. and McDonough, F. M., EPA's Proposed Academic Laboratories Rule: A More Flexible Approach to the Management of Hazardous Waste. Journal of Chemical Health \& Safety 13: 5-8, 2006.

[5] US Environmental Protection Agency., Waste Minimization Opportunity Assessment Manual, Publication EPA/625/7-88/003, Hazardous Waste Engineering Research Laboratory: Cincinnati, 1998.

[6] Pipitone, D. A., Safe Storage of Laboratory Chemicals, 2nd ed, John Wiley \& Sons: USA, 1991.

[7] US Environmental Protection Agency., Guides to Pollution Prevention, Research and Educational Institutions, Government Printing Office: Washington, DC, 1990.

[8] American Chemical Society., The Waste Management Manual for Laboratories, ACS, Dept. of Government Relations and Science Policy: Washington, USA, 1990.

[9] Luxon, S. G., Hazards in the Chemical Laboratory, 5th ed, Royal Society of Chemistry: Cambridge, United Kingdom, 1992.

[10] Environmental Health and Safety Hazardous Waste Minimization Plan, University of California, Santa Barbara: USA, 2007. Online http://www.ehs.ucsb.edu/units/hw/hwrsc/hwpdf/HazWaste_Minimization Plan.pdf.

[11] Hazardous Waste Disposal Procedures, Safety Manual, University of Pittsburgh. USA, 2010. Online http://www.ehs.pitt.edu/assets/docs/ HazardousWaste.pdf.

[12] Hill, D. C., Establishing a Waste Minimization Program. Professional Safety 8: 16, 1992.

[13] Drew University Hazardous Waste Management Plan. South Carolina, HRP Associates, Inc., USA, 2011, Online http://www.drew.edu/wp-content/ uploads/sites/62/Drew-University-Haz-Waste-Management-PlanDecember2011.pdf.

[14] American Chemical Society., Less is Better: A Guide to Minimizing Waste in Laboratories, American Chemical Society, National Academy Press: 2002, USA.

[15] National Research Council., Prudent Practices for Disposal of Chemicals from Laboratories, National Academy Press: USA, 1983. 